\title{
Analysis of Dynamic Behavior of a Class of Nonlinear Systems Subject to Disturbances
}

\author{
Wang Haiying \\ Department of Information and computation science \\ Chongqing University \\ Chongqing,China \\ e-mail: xiongying200818@sohu.com
}

\author{
Wang Disheng \\ Department of Computer \\ Chengdu College of University of Electronic Science \\ and Technology \\ Chengdu,China \\ e-mail: 2328552759@qq.com
}

\begin{abstract}
Nonlinearity is an important characteristic of complex system, is generally believed that the nonlinearity is a necessary condition to produce complexity, no nonlinear no complexity. In addition, bifurcation, chaos in complex system, the dynamic behavior of the strange attractor, are inherently nonlinear. The nonlinear dynamic behavior, often by a certain amount (or large) nonlinear element (or subsystems) due to the combination and interaction, is essentially different from the linear element combination and interaction. In fact, the nonlinear factors in complex systems (internal and environmental) and their interactions is an important condition for the formation of complexity. Financial power system is a complex nonlinear system is a kind of typical, it may exhibit a variety of complex phenomenon and behavior, including chaos, there may be many disturbance factors in the actual system, disturbance exists from the impact of various aspects of the stability of the whole financial system, and even the emergence of the financial crisis. In this paper, through the establishment of nonlinear financial power system model with perturbations, using the theory of differential dynamical system, discussed the system stability and chaos, get the system stability conditions. The economic implications are given in the end.
\end{abstract}

Keywords- The nonlinear;time delay; stability;chaos;

financial

\section{INTRODUCTION}

Nonlinearity is an important characteristic of complex system, is generally believed that the nonlinearity is a necessary condition to produce complexity, no nonlinear no complexity. In addition, bifurcation, chaos in complex system, the dynamic behavior of the strange attractor, are inherently nonlinear. The nonlinear dynamic behavior, often by a certain amount (or large) nonlinear element (or subsystems) due to the combination and interaction, is essentially different from the linear element combination and interaction. In fact, the nonlinear factors in complex systems (internal and environmental) and their interactions is an important condition for the formation of complexity. The Financial System is a typical complex system, the relationship between the factors system of internal and external are usually nonlinear, and the nonlinear system possible instability and complexity in the process of operation, may cause the entire economic system instability even in the external conditions of certain small changes occur, the precise economic prediction is very limited, the expected behavior of a reasonable can become more complicated,for example, between supply and demand, fluctuations in the economic cycle, monetary shortage, information asymmetric; consumer behavior "limited rationality" and so on. The nonlinear system is not only a kind of system in accordance with the principle of superposition, it can lead to catastrophe. The financial system and risk management has been very concerned about the social from all walks of life problems. It is necessary to study the academic and financial system behavior to find the rules and characteristics,.

In th paper we consider in the state variable disturbances, behavior characteristics and laws of the financial system, the economic implications are given in the end.

\section{FINANCIAL POWER SYSTEM MATHEMATICAL MODEL}

Literature [1] using system dynamics method to build and test a production that block, block, currency financial model composed of securities sub block and labor sub block, sensitivity to certain long-term behavior of this model is given with no rules and parameters of the initial state of the extreme, in order to further study the reason and mechanism the chaotic behavior patterns, people will be the key part in the model to be refined and simplified, after careful analysis and experiments, decided to model in the interest rate is represented by the variable $x$, investment demand is represented by the variable $y$, price index is represented by the variable $Z$. The three state variable and included in their information feedback loop as the key structure. By selecting the appropriate coordinate system and give the state variable to the appropriate dimension, obtained the following contains only 3 main parameters of the simpler model.

Financial power system model:

$$
\left\{\begin{array}{l}
\dot{x}=z+(y-a) x \\
\dot{y}=1-b y-x^{2} \\
\dot{z}=-x-c z
\end{array}\right.
$$


The type $\mathrm{x}$ is the rate of interest, $\mathrm{y}$ said that investment demand, says $z$ price index, a savings, $b$ is the unit investment cost, c for commodity demand elasticity (a, b, c were normal number).

Now consider the description of disturbance factors for:

$$
\left\{\begin{array}{l}
\dot{x}=\sigma(x-y) \\
\dot{y}=r x-y-x z \\
\dot{z}=x y-s z
\end{array}\right.
$$

Explain the economic implications:

The first equation expressed interest rate with the time response is affected by the rate of interest and the demand for investment, here assumed to affect the strength, the difference between the two or the asymmetric information, it will cause interest rate changes with the step of time;

The second equations can be expressed in making investment decisions or management stage, decided to jointly the effect of investment demand from the early stage of interest rate, the investment demand and the interest rate and price index.

Third expression is the price index trend, can be regarded as the market reaction after the market function and management decision control. Interaction of price index and the interest rate and investment demand is positive correlation, and the price index growth which shows a certain corrosion tendency.

Will (1) type power system into without constant term, The system also can get to replace the original equilibrium point $P_{0}$ of origin $(0,0,0)$, The following expressions:

$$
\left\{\begin{array}{l}
\dot{x}_{1}=(1 / b-a) x_{2}+x_{3}+x_{1} x_{2} \\
\dot{x}_{2}=-b x_{2}-x_{1}^{2} \\
\dot{x}_{3}=-x_{1}-c x_{3}
\end{array}\right.
$$

Will (3) to (2) in the

right,let $x \rightarrow x_{1}, \mathrm{y} \rightarrow x_{2}, z \rightarrow x_{3}$, we get:

Financial power system with risk factors:

$$
\left\{\begin{array}{l}
\dot{x}_{1}=\sigma x_{1}+(1 / b-a-\sigma) x_{2}+x_{3}+x_{1} x_{2} \\
\dot{x}_{2}=r x_{1}+(-b-1) x_{2}-x_{1}^{2}-x_{1} x_{3} \\
\dot{x}_{3}=-x_{1}-(c+s) x_{3}+x_{1} x_{2}
\end{array}\right.
$$

Financial power system disturbance with risk factors:

$$
\left\{\begin{array}{l}
\dot{x}_{1}=(\sigma+\delta \sigma) x_{1}+(1 / b-a-\sigma-\delta \sigma) x_{2}+x_{3}+x_{1} x_{2} \\
\dot{x}_{2}=(r+\delta r) x_{1}+(-b-1) x_{2}-x_{1}^{2}-x_{1} x_{3} \\
\dot{x}_{3}=-x_{1}-(c+s+\delta s) x_{3}+x_{1} x_{2}
\end{array}\right.
$$

The type $\mathrm{x}$ is the rate of interest, $\mathrm{y}$ said that investment demand, says z price index, a savings, $b$ is the unit investment cost, c for commodity demand elasticity, $\sigma$ said the expansion coefficient of asymmetric effect; $r$ The times leverage effect management regulation on investment demand; $s$ said the price index of the current price index weakening ratio, The disturbance of system parameters: $\delta \sigma, \delta r, \delta s$

\section{ANALYSIS OF DYNAMIC SYSTEMS}

\section{A. Analysis of dynamic characteristics}

In system (1):When $c-b-a b c \leq 0$, there is a only equilibrium point $P_{0}(0,1 / b, 0)$, and then $c-b-a b c<0,1+a c-c / b>0$, the equilibrium point $P_{0}$ is stable. When $c-b-a b c<0$ $c+a-1 / b<0$, the equilibrium $P_{0}$ is a saddle point. When $c+a-1 / b=0,0<\mathrm{c}<1$, the equilibrium point $P_{0}$ is non-hyperbolic and unstable equilibrium point. When $c-b-a b c \geq 0$, the system has three equilibrium points: $P_{0}(0,1 / b, 0) P_{1,2}( \pm \theta,(1+a c) / c, \mp \theta / c)$, When $c-b-a b c=0,0<c<1, P_{0}$ is the unstable equilibrium point; When $c>b+a b c$, when the parameters satisfy $b c^{4}+b^{2} c^{3}-2 a b^{2} c^{2}+\left(2 a b-2-3 b^{2}\right) c+3 b=0$ the system (1) appears bifurcation, $P_{1,2}$ an unstable equilibrium point.

Take parameters $\mathrm{a}=0.9, \mathrm{~b}=0.2, \mathrm{c}=1.2$, the initial conditions [3, 1, 5],to simulate by using Matlab software, when the simulation time was set to $[0,10]$ and $[0,1000]$, get the three-dimensional phase diagram of finance chaotic system (1) as shown in Fig .1, Fig .2, respectively. Fig .1, Fig .2 shows that as time extending, the chaotic behavior of the economic system become more and more prominent.

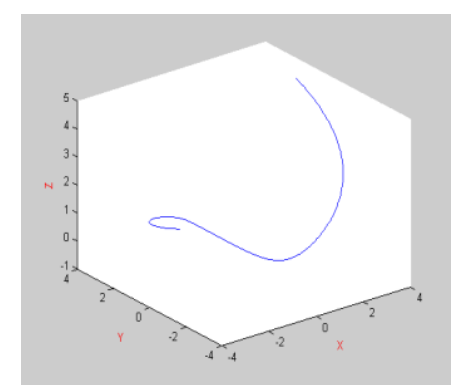

Figure 1. the three-dimensional phase diagram when simulation time is $[0,10]$ 


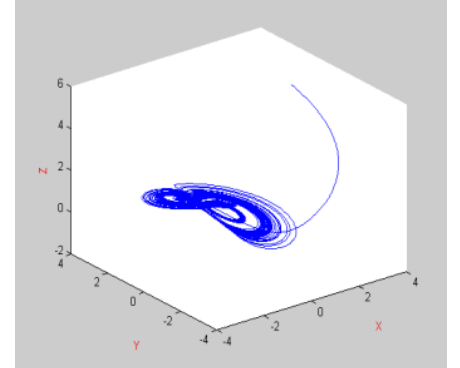

Figure 2. the three-dimensional phase diagram when simulation time is $[0,1000]$

By the above analysis and data simulation shows that the inappropriate combination of parameters in the system cause the system to the source of chaos, it is possible to make the system tend to chaotic and out of control, resulting in economic turmoil, when the parameters will appear in a different system state,analysis of changes in various parameters to get the kind of financial system of local conditions to produce complex behavior.

\section{B. Analysis of Dynamic Systems with disturbance with risk factors}

a) Singular case

Make (5) type with zero on the right, Available for singular point of origin $\mathrm{O}(0,0,0)$

The system is written as follows:

$$
\left\{\begin{array}{l}
\dot{x}_{1}=(\sigma+\delta \sigma) x_{1}+(1 / b-a-\sigma-\delta \sigma) x_{2}+x_{3}+\alpha\left(x_{1}, x_{2}, x_{3}\right) \\
\dot{x}_{2}=(r+\delta r) x_{1}+(-b-1) x_{2}+\beta\left(x_{1}, x_{2}, x_{3}\right) \\
\dot{x}_{3}=-x_{1}-(c+s+\delta s) x_{3}+\chi\left(x_{1}, x_{2}, x_{3}\right)
\end{array}\right.
$$

Among them:

$$
\begin{aligned}
& \alpha\left(x_{1}, x_{2}, x_{3}\right)=\chi\left(x_{1}, x_{2}, x_{3}\right)=x_{1} x_{2} \\
& \beta\left(x_{1}, x_{2}, x_{3}\right)=-x_{1}^{2}-x_{1} x_{3}
\end{aligned}
$$

Obviously,

$$
\alpha\left(x_{1}, x_{2}, x_{3}\right), \beta\left(x_{1}, x_{2}, x_{3}\right), \chi\left(x_{1}, x_{2}, x_{3}\right)
$$

In the $\mathrm{O}(0,0,0)$ neighborhood of a differentiable,

And to meet the:

$$
\lim _{\substack{x_{1} \rightarrow 0 \\ x_{2} \rightarrow 0 \\ x_{3} \rightarrow 0}} \frac{\alpha\left(x_{1}, x_{2}, x_{3}\right)}{\sqrt{x_{1}^{2}+x_{2}^{2}+x_{3}^{2}}}=\lim _{\substack{x_{1} \rightarrow 0 \\ x_{2} \rightarrow 0 \\ x_{3} \rightarrow 0}} \frac{\beta\left(x_{1}, x_{2}, x_{3}\right)}{\sqrt{x_{1}^{2}+x_{2}^{2}+x_{3}^{2}}}=\lim _{\substack{x_{1} \rightarrow 0 \\ x_{2} \rightarrow 0 \\ x_{3} \rightarrow 0}} \frac{\chi\left(x_{1}, x_{2}, x_{3}\right)}{\sqrt{x_{1}^{2}+x_{2}^{2}+x_{3}^{2}}}=0
$$

Therefore, we can study the system (5) and approximate linear system:

$$
\left\{\begin{array}{l}
\dot{x}_{1}=(\sigma+\delta \sigma) x_{1}+(1 / b-a-\sigma-\delta \sigma) x_{2}+x_{3} \\
\dot{x}_{2}=(r+\delta r) x_{1}+(-b-1) x_{2} \\
\dot{x}_{3}=-x_{1}-(c+s+\delta s) x_{3}
\end{array}\right.
$$

type of singularity and stability of linear system to study the singular points of the system type and the zero solution in the neighborhood of the stability of the system.
The coefficient matrix $A$ of system (7)for:

$$
A=\left(\begin{array}{ccc}
\sigma+\delta \sigma & 1 / b-a-\sigma-\delta \sigma & 1 \\
r+\delta r & -b-1 & 0 \\
-1 & 0 & -(c+s+\delta s)
\end{array}\right)
$$

When

$|A|=(c+s+\delta s)[(\sigma+\delta \sigma)(b+1)+(\mathrm{r}+\delta r)]-(b+1)=0$ is the system of higher singularity;

When $|A| \neq 0, \mathrm{O}(0,0,0)$ is the elementary singular point linear approximation system.

b) Stability

The above formula (7) for the characteristic equation coefficient matrix:

$$
|A-\lambda I|=\left|\begin{array}{ccc}
\sigma+\delta \sigma-\lambda & 1 / b-a-\sigma-\delta \sigma & 1 \\
r+\delta r & -b-1-\lambda & 0 \\
-1 & 0 & -(c+s+\delta s)-\lambda
\end{array}\right|=0
$$

That is:

$$
\begin{aligned}
& \lambda^{3}-\lambda^{2}(\sigma+\delta \sigma-s-\delta s-b-c-1)-\lambda[(\sigma+\delta \sigma)(\mathrm{b}+1)+(\mathrm{r}+\delta \mathrm{r})(c+s+\delta s)-1] \ldots \\
& \cdots-(c+s+\delta s)[(\sigma+\delta \sigma)(b+1)+(\mathrm{r}+\delta r)]-(b+1)=0
\end{aligned}
$$

Using the Routh-Hurwitz criteria, there is:

$$
|A-\lambda I|=a_{0} \lambda^{3}+a_{1} \lambda^{2}+a_{2} \lambda+a_{3}=0
$$

Among them $a_{0}>0$,sructural determinant:

$$
\Delta_{3}=\left(\begin{array}{lll}
a_{1} & a_{0} & 0 \\
a_{3} & a_{2} & a_{1} \\
a_{5} & a_{4} & a_{3}
\end{array}\right)
$$

In the formula, the linear approximation system type (7) the characteristic equation of all roots have negative real part,its necessary and sufficient conditions are all the principal minors of a $\Delta_{3}$ are greater than zero, That is:

$$
\begin{gathered}
\Delta_{2}=\left|\begin{array}{c}
\Delta_{1}=1>0 \\
-(\sigma+\delta \sigma-s-\delta s-b-c-1) \\
-(c+s+\delta s)[(\sigma+\delta \sigma)(b+1)+(\mathrm{r}+\delta r)]-(b+1) \\
1 \\
-\lambda[(\sigma+\delta \sigma)(\mathrm{b}+1)+(\mathrm{r}+\delta \mathrm{r})(c+s+\delta s)-1]
\end{array}\right|>0 \\
\Delta_{3}=\left|\begin{array}{c}
\ldots \\
-(c+s+\delta s)[(\sigma+\delta \sigma)(b+1)+(\mathrm{r}+\delta r)]-(b+1) \ldots \\
0 \\
1 \\
\ldots-\lambda[(\sigma+\delta \sigma)(\mathrm{b}+1)+(\mathrm{r}+\delta \mathrm{r})(c+s+\delta s)-1] \ldots \\
0 \\
0 \\
\quad \ldots \quad-(c+s+\delta s)[(\sigma+\delta \sigma)(b+1)+(\mathrm{r}+\delta r)]-(b+1)
\end{array}\right|>0
\end{gathered}
$$

The above formula are established,the zero solution is asymptotically stable. 
Conclusion: When combinations of parameters system to meet $, \Delta_{i}>0 \quad i=1,2,3$ the zero solution is asymptotically stable.

\section{ENDING}

Get some inspiration from the previous analysis:

First, combination of each parameter improper system is caused by the economic system appear chaos roots, it is possible to make the system tends to chaos and out of control, there may be a system of stagnation rigid state, therefore, whether in the period of inflation is serious, or in the period of economic recession, the conversion mechanism, structural adjustment is an important task for finance system.

Second, lack of elastic variables will cause the delay information feedback, only error signal given the decision-making body of the distorted, adverse consequences arising therefrom is one can imagine, what is more serious is that these adverse consequences will be to the vicious spiral form continues to spread. Therefore, properly increasing the variable elastic will help to stabilize the economy, the normal operation of the financial system.

Third, the amount of storage system variables must be maintained at an appropriate level. The results of the study show that, smaller, the larger fluctuation, too hours can lead to chaos of the situation; however, it can not be too large, otherwise, will make the economy less energy.

Fourth, different combinations of different parameters in the system can lead to the bifurcation phenomenon, bifurcation represents the transition between economic growth during different growth pattern.

Fifth, if the selection of appropriate control strategy and direction, a good grasp of financial control, power system can be stabilized.

Need to explain is: Although nonlinear science have made great progress in all areas, but compared to natural science, its research results achieved in the field of economics, finance limited. The nonlinear dynamics method applied to economics, a master key is not the solution to all problems. In the field of finance, equity and social economy due to the interaction of nonlinear factors, economic problems become more and more complicated and from low - to high dimensional evolution process, the internal structure of system is also showing a diversity and complexity, therefore, further study on intrinsic structural features of these systems will, through the cycle of the system the solution of the instability, bifurcation, period doubling bifurcation, the bifurcation point, the complex system into chaos and so on, to reveal the causes of complex phenomena, and on this basis, to provide the theoretical basis and practical method for the analysis of complex continuous economic system. In short, there are still a lot of work to do.

\section{REFERENCE}

[1] Huang Dengshi, Li Houjiang, Nonlinear economics theory and method ,Chengdu: university of Sichuan press, 1993,55-60.
[2] Li Zushu, Tu Yaqing, Human-simulated intelligent control,Beijing: National defense industry press,2003.

[3] LiuBao,Modern control theory,Beijing: Mechanical industry press,2002,149-150.

[4] Fadhil Rahma, Ramzy S. Ali, Luigi Fortuna, Mattia Frasca, "New Chaotic Attractors and New Chaotic Circuits," IJACT, Vol. 4, No. 3, pp. $125 \sim 132,2012$.

[5] Ling Ling Song, "Navigation Control of an Autonomous Robot Based on Chaos Immune Optimization Algorithm, ” AISS, Vol. 4, No. 9, pp. $302 \sim 310,2012$.

[6] Wenyuan Zhang, Yunfei Ma, Gang Yang, "Study on Parameter Selection of Phase Space Reconstruction for Chaotic Time Series, "AISS, Vol. 4, No. 2, pp. 67 77, 2012.

[7] Zhang Xiaodan,Li Zhiping,Zhang Lili, "A class of singular value decomposition calculation based on Lyapunov index," Journal of University of Science and Technology Beijing, vol. 27 (3),2005, pp. 50-58.

[8] Wei-Ching Chen, "Dynamics and control of a financial system wih time-delayed feedbacks, " Chaos, Solitons and Fratals, vol. 37,2008,pp.1198-1207.

[9] Carl Chiarella, Xue-Zhong He, "A dynamic analysis of moving average rules, " Journal of Economic Dynamics\& Control, vol. 30,2006,pp.1729-1753.

[10] Xiaoling Ke, “Ke shi stability and bifurcation in a simple heterogeneous asset pricing model, " Economic Modeling, vol. 26,2009, pp.680-688.

[11] Xue-Zhong He, "Market stability switches in a continuous-time financial market with heterogeneous beliefs, " Economic Modeling,vol. 26 ,2009, pp.1432-1442.

[12] Shen J,Zhen G B, Lin H, et al, "Dynamic Relax at ion of Financial Indices," Modern Physics Letters B, vol.23,2009,pp. 2889 2897.

[13] Yuan Lili, "Mathematical finance development of goods and quality," theory research, vol. 7,2011,pp. 117.

[14] Jiao Hongbing, "Properties of fixed asset investment model with solutions of delay," System science and Mathematics, vol. 20 (1), 2000,pp. 14-23.

[15] Zhang Zhuanzhou, et al, "Dynamic analysis of a new threedimensional nonlinear system," Applied mathematics and mechanics, vol. 34 (12) ,2013,pp. 1321-1326. 